\title{
A Water-Dispersible Quinoid-Resonant Conducting Polymer for Organic Electronics
}

\author{
Dafei Yuan $^{a, b}$ (D) \\ Xiaozhang Zhu*a,b (i)

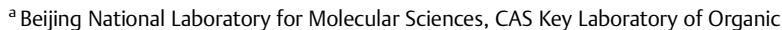 \\ Solids, Institute of Chemistry, Chinese Academy of Sciences, Beijing 100190, P. R. China \\ ${ }^{\mathrm{b}}$ School of Chemical Sciences, University of Chinese Academy of Sciences, Beijing \\ 100049 , P. R. China. \\ xzzhu@iccas.ac.cn
}

\section{Received: 11.05 .2020}

Accepted after revision: 4.06.2020

DOI: 10.1055/s-0040-1714145; Art ID: om-20-0016sc

License terms: (c)

(c) 2020. The Author(s). This is an open access article published by Thieme under the terms of the Creative Commons Attribution-NonDerivative-NonCommercial-License, permitting copying and reproduction so long as the original work is given appropriate credit. Contents may not be used for commercial purposes, or adapted, remixed, transformed or built upon. (https://creativecommons.org/licenses/by-nc-nd/4.0/).

Abstract Developing stable and solution-processable highly conductive polymers has been the research goal in organic electronics since the first demonstration of metallic conductive polyacetylene. Here, we used a unique quinoid-resonant building block thieno[3,4-b]thiophene (TbT) to develop a new water-dispersible conducting polymer, PTbT-Me:PSS. Linear polymerization and large surfactant counterion, poly(styrenesulfonate) (PSS ${ }^{-}$), were introduced, which enabled a high electrical conductivity of $68 \mathrm{~S} \mathrm{~cm}^{-1}$ and exhibited water-dispersible property. Interchain bipolaron was found in PTbT-Me:PSS when compared with polaron in PEDOT:PSS in their conducting mechanism. Moreover, we applied this highly conductive PTbT-Me:PSS as the solution-processed polymer thermoelectric material and a decent power factor of $3.1 \mu \mathrm{W}$ $\mathrm{m}^{-1} \mathrm{~K}^{-2}$ was achieved.

Key words conducting polymers, solution-processed, electronic devices, organic thermoelectric materials, energy conversion

\section{Introduction}

Organic conducting polymers exhibit great potential in electronic markets because these conductors are able to be solution-processed, flexible, light-weight, low-cost, and chemically modified. ${ }^{1}$ The milestone of conducting polymers is that the insulating polyacetylene (PA) films exhibit metallic behavior with an electrical conductivity exceeding $10^{5}$ $\mathrm{Scm}^{-1}$ after doping with iodine. ${ }^{2}$ The combination of flexibility and high conductivity makes PA a good candidate for organic electronics. However, the poor solubility and low stability of doped PA greatly limit its practical application. During the recent decades, scientists have focused on developing ambient-stable and solution-processed conducting polymers. ${ }^{3}$ However, only several conducting polymers, for example,
Quinoid-Resonant Water-Dispersible Conducting Polymers

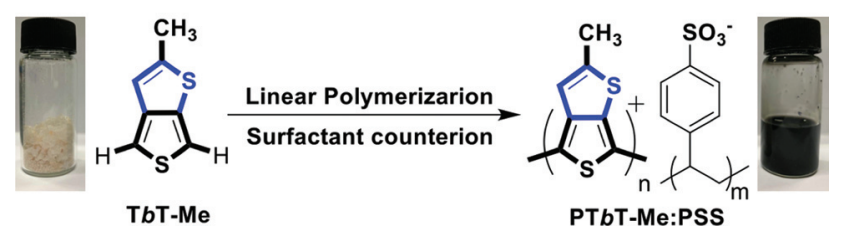

polyaniline (PANI), ${ }^{3 \mathrm{a}, 3 \mathrm{~b}, 4}$ poly(3,4-ethylenedioxythiophene) (PEDOT), ${ }^{5}$ etc. based conducting polymers, meet the demands and become commercial available. PEDOT holds a rigid and planar conjugated polymer backbone, which facilitates charge transport in charged states and is extensively investigated. Very recently, sequential solution polymerization ${ }^{6}$ and selfdoping ways ${ }^{7}$ have been developed to further improve the chemical and electrical properties of PEDOT. The wide range of electrical conductivity and especially the good solubility in an environmental-friendly solvent (water) of the commercially available PEDOT:PSS enable various modern electronic applications, for example, conducting layer for organic field-effect transistors ${ }^{8}$ and electrochromic devices, ${ }^{9}$ hole transporting layer for photovoltaics (OPVs), ${ }^{10}$ and light emitting diodes (OLEDs), ${ }^{11}$ antistatic layers for photographic films, ${ }^{12}$ etc. More recently, PEDOT has become attractive in organic spintronics and organic polymer thermoelectrics, in which long spin lifetimes and high thermoelectric efficiencies are achieved. ${ }^{13}$ However, the limitedly tunable chemical property in PEDOT impedes the further investigation and application. Developing new organic conducting material systems has become emergent and essential.

Polythieno[3,4-b]thiophene (PT $b \mathrm{~T}$ ) has been prepared and systematically investigated since the last two decades. ${ }^{14}$ The unique quinoid-resonant effect in $\mathrm{T} b \mathrm{~T}$ can efficiently narrow the bandgap and hold rigidity and planarity of the backbone, ${ }^{15}$ which is promising in developing highly conductive organic conductors. Besides, the rich chemical tunability in PTbT, in comparison with PEDOT, makes it more attractive in organic electronics. However, the previous report on PTbT shows low electrical conductivity and poor solubility. ${ }^{14 a, 14 b, 14 c}$ The first water-dispersible PTbT-C0-PSS (Scheme 1) was prepared by Sotzing et al. in $2005,{ }^{14 \mathrm{~d}}$ by oxidative polymerization of $\mathrm{TbT}$ monomer (TbT-CO) with $\mathrm{Fe}^{3+}$ as an oxidant and poly (styrenesulfonate) $\left(\mathrm{PSS}^{-}\right.$) as a counterion. Because of more than two reactive sites on TbT-C0 monomers, nonlinear polymerization works in the polymerization and leads to the network-like homo-polymers. Although the PTbT-C0:PSS thin film shows an electrical conductivity of $0.02 \mathrm{~S} \mathrm{~cm}^{-1}$ under ambient conditions, further enhancement of electrical conductivity is necessary. Recently, we propose linear 


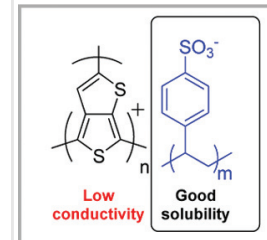

PTbT-C0:PSS

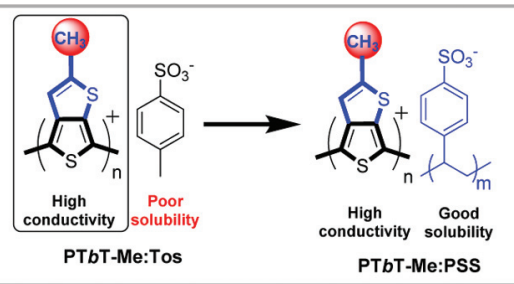

PTbT-Me:PSS
Scheme 1 Molecular structures of PTbT-C0:PSS, PTbT-Me:Tos, and PTbT-Me:PSS.

polymerization and alkyl chain engineering for PTbT to enable high electrical conductivity $\left(450 \mathrm{~S} \mathrm{~cm}^{-1}\right)$ and excellent thermoelectric performance in PTbT-Me:Tos, which is even superior to PEDOT:Tos under the same conditions (Scheme 1). ${ }^{14 \mathrm{f}}$ Because the 2-position methyl (-Me) terminal group-substituted $\mathrm{T} b \mathrm{~T}$ monomer (TbT-Me) and smaller counterion, tosylate $\left(\operatorname{Tos}^{-}\right)$, that coupled to the PTbT backbone are used for the solution-cast polymerization, the solubility is limited in this highly conductive polymer. ${ }^{14 \mathrm{f}}$

To enable good solubility and maintain high conductivity in PTbT, we report in this work the preparation of the water-dispersible conducting polymer PTbT-Me:PSS by the combination of linear polymerization with highly conductive PTbT-Me as the conjugated backbone and large waterdispersed surfactant PSS $^{-}$as the counterion (Scheme 1). PTbT-Me:PSS can disperse in water and this conducting solution can be stored over several weeks under ambient conditions. The PTbT-Me:PSS thin film showed an excellent electrical conductivity of $68 \mathrm{~S} \mathrm{~cm}^{-1}$, nearly 4 orders of magnitude higher than the previously reported PTbT-C0: PSS. $^{14 \mathrm{~d}}$ Besides, in comparison with polaron-dominant PEDOT:PSS and PTbT-Me:Tos that showed paramagnetism, ${ }^{13 c, 14 f}$ PTbT-Me:PSS exhibited diamagnetism and interchain bipolaron-dominant hopping behavior. ${ }^{16}$ We further applied this conducting polymer for thermoelectric application, in which a power factor of $3.1 \mu \mathrm{W} \mathrm{m} \mathrm{m}^{-1} \mathrm{~K}^{-2}$ was achieved by the PTbT-Me:PSS thin film at room temperature.

\section{Results and Discussion}

TbT-Me was synthesized according to the reported procedures as white powder in a yield of $60 \%$. TbT-Me showed moderate stability in air, but can be stored at $-10{ }^{\circ} \mathrm{C}$ in a dark place for weeks. ${ }^{14 \mathrm{e}}$ According to the previous study, ammonium persulfate $\left[\left(\mathrm{NH}_{4}\right)_{2} \mathrm{~S}_{2} \mathrm{O}_{8}\right]$, hydrogen peroxide $\left(\mathrm{H}_{2} \mathrm{O}_{2}\right)$, and ferric sulfate $\left[\mathrm{Fe}_{2}\left(\mathrm{SO}_{4}\right)_{3}\right]$ were used as oxidants for polymerization of TbT and the PTbT-CO:PSS thin film from $\left[\mathrm{Fe}_{2}\left(\mathrm{SO}_{4}\right)_{3}\right]$ oxidative polymerization gave the best electrical conductivity. ${ }^{14 \mathrm{~d}}$ Therefore, we used $\mathrm{Fe}_{2}\left(\mathrm{SO}_{4}\right)_{3}$ as the oxidant in this work. As shown in Scheme S1, Figure S1,

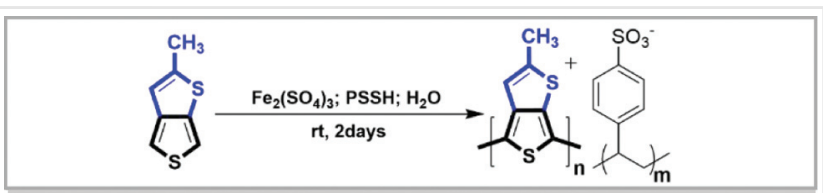

Scheme 2 Preparation of conducting polymer, PTbT-Me:PSS.

and Scheme $2,{ }^{14 \mathrm{~d}}$ after mixing the TbT-Me monomer, $\mathrm{Fe}_{2}\left(\mathrm{SO}_{4}\right)_{3}$, and $\mathrm{PSSH}$ in deionized water, the mixture was stirred at room temperature in air for 2 days with the solution color changing from white to grey, and then black. ${ }^{17}$ The insoluble precipitate was leached by a filter membrane and a black solution was obtained as shown in Figure S1. The PTbT-Me:PSS thin film was prepared by spin-coating the polymer solution on a precleaning substrate and then transferred onto a hot-plate for thermal annealing.

The electrical conductivities of PTbT-Me:PSS films were determined by a standard four-probe method. As shown in Figure S2a, the electrical conductivity of the PTbT-Me:PSS film was determined to be $0.15 \mathrm{~S} \mathrm{~cm}^{-1}$ at a low oxidant level (molar ratio, $\mathrm{Fe}^{3+}: \mathrm{T} b \mathrm{~T}-\mathrm{Me}=0.5: 1$ ) and then increased to $0.22 \mathrm{~S} \mathrm{~cm}^{-1}\left(\mathrm{Fe}^{3+}: \mathrm{T} b \mathrm{~T}-\mathrm{Me}=2: 1\right)$. Through optimization of mass ratio of PSSH and TbT-Me (Figure S2b), the highest electrical conductivity of $0.6 \mathrm{~S} \mathrm{~cm}^{-1}$ was achieved under ambient conditions, when the weight ratio of PSSH:TbT-Me was 7.2:1. After optimizing the thermal-annealing temperature and preparing the PTbT-Me:PSS thin film under a $\mathrm{N}_{2}$ atmosphere (Figure S3), the electrical conductivity was significantly enhanced to $5 \mathrm{~S} \mathrm{~cm}^{-1}$.

The excess of PSSH was used for polymerization, which not only acted as counterion in PTbT-Me:PSS but also impeded the charge transport because it was insulating. De-doping with high-boiling point solvents to remove the excess of PSSH has been proved to be effective to improve electrical conductivity and achieve excellent thermoelectric performance as shown in the case of PEDOT:PSS. ${ }^{13 \mathrm{~b}}$ Therefore, in this work, we used a hydrophilic and high-boiling point solvent, ethylene glycol(EG), to selectively de-dope the excess insulating PSS $^{-}$. As shown in Figure 1a, after EG treatment for 10 minutes at $60{ }^{\circ} \mathrm{C}$ under a $\mathrm{N}_{2}$ atmosphere, the film thickness of PTbT-Me:PSS decreased significantly from around 180 to $130 \mathrm{~nm}$, which implied that part of PSS was removed. With further EG treatment, the film thickness decreased slowly to $115 \mathrm{~nm}$ and almost remained constant with even longer treatment time. Accordingly, the electrical conductivity of PTbT-Me:PSS films increased dramatically from 5 to $60 \mathrm{~S} \mathrm{~cm}^{-1}$ after EG treatment for 10 minutes (Figure 1b). After that, the electrical conductivity increased only slightly from 60 to $68 \mathrm{~S} \mathrm{~cm}^{-1}$ in consistent with the changes of film thickness. The electrical conductivity achieved in the PTbT-Me:PSS film was 3 orders of magnitude 

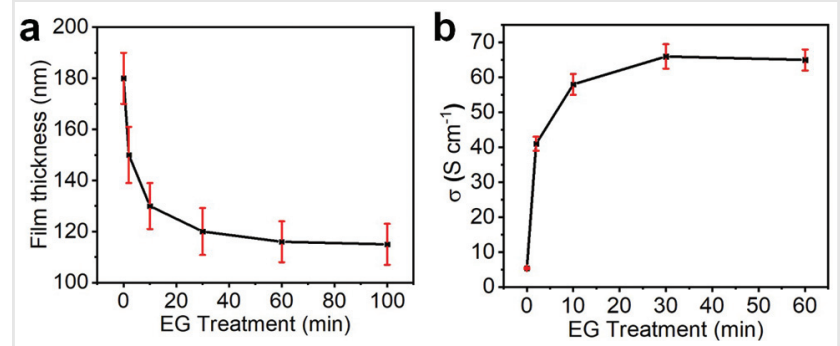

Figure 1 (a) Film thickness and (b) electrical conductivity changes of PTbT-Me:PSS films with EG treatment.

higher than the reported network-like conducting polymer, PTbT-C0-PSS $\left(0.02 \mathrm{~S} \mathrm{~cm}^{-1}\right)$, under the same conditions, demonstrating that linear-polymerization was important in developing highly conductive T $b \mathrm{~T}$ polymers.

To investigate the de-doping process, ultraviolet-visiblenear-infrared spectroscopy (UV-Vis-NIR), X-ray photoelectron spectroscopy (XPS), and ultraviolet photoelectron spectroscopy (UPS) were conducted on the pristine and de-doped films with different EG treatment times. As shown in Figure 2a, a weak absorption in the visible region but a strong chargeresonance absorption band extending over $2500 \mathrm{~nm}$ was found in the pristine conducting polymer thin film, implying that charge carriers can be delocalized on the PTbT backbone. ${ }^{14 f, 18}$ After EG treatment, a slightly increased absorption intensity in the region of 750-1750 nm was found in de-doped films. As shown in the XPS spectra of sulfur (2p), the peak at the binding energy of 172-166 eV was assigned to the sulfur of PSS $^{-}$, while the peak at the binding energy of $166-162 \mathrm{eV}$ was assigned to the sulfur of PTbT (Figure 2b). ${ }^{14 f, 19}$ By calculating the integral areas of sulfur peak intensity on $\mathrm{PSS}^{-}$and PT $b \mathrm{~T}$, the area of sulfur on PSS $^{-}$was around nine times larger than the
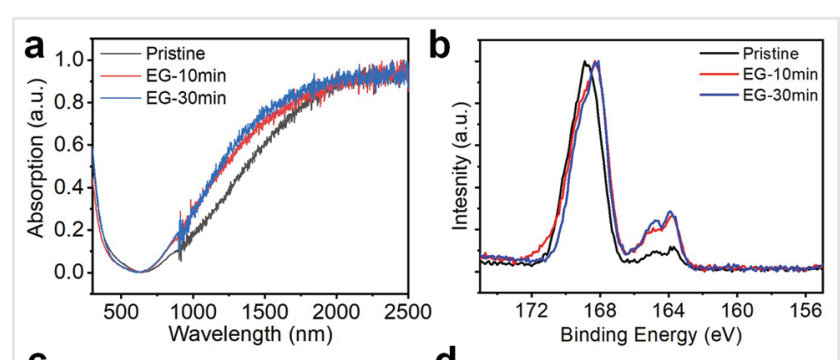

C
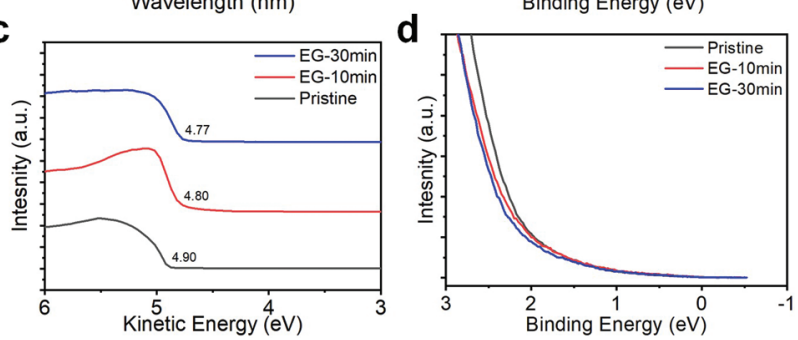

Figure 2 (a) UV-Vis-NIR, (b) S (2p) XPS (normalization based on the peak of $\mathrm{S}$ on $\mathrm{PSS}^{-}$), (c) the low kinetic energy region and (d) the low binding energy region (HOMO) of UPS spectra. sulfur peak on PTbT ( $\left.\mathrm{S}_{\mathrm{PSS}}: \mathrm{S}_{\mathrm{PT} b \mathrm{~T}}=9.25: 1\right)$, which (ratio) was much higher than the commercial PEDOT:PSS ( $\mathrm{S}_{\mathrm{PSS}}: \mathrm{S}_{\mathrm{PEDOT}} \approx$ 2:1, PH 1000), ${ }^{13 \mathrm{~b}}$ indicating that much excess of insulating PSS $^{-}$existed in the PTbT-Me:PSS film without coupling with the positive charge on the PTbT polymer chain. This large amount of insulating PSS ${ }^{-}$will wrap the PTbT conjugated backbone and impede charge transport that is consistent with the low conductivity of the pristine PTbT-Me:PSS film. ${ }^{20}$ After treatment with EG for 10 minutes, the integral area of the sulfur peak on PTbT significantly increased relative to PSS $^{-}$and the ratio decreased to $\mathrm{S}_{\mathrm{PSS}}: \mathrm{S}_{\mathrm{PT} b \mathrm{~T}}=4.15: 1$ as shown in Figure 2b, suggesting that nearly half of the excess PSS $^{-}$ was removed. Further treatment with EG, the ratio of $S_{\mathrm{PSs}}: \mathrm{S}_{\mathrm{PT} T \mathrm{~T}}$ decreased slightly, demonstrating that the remaining PSS $^{-}$ interacted strongly with PTbT and was difficult to remove by hydrophilic solvents. Therefore, the film thickness and electrical conductivity of PTbT-Me:PSS ${ }^{-}$changed slightly after EG treatment over 30 minutes. As depicted in the low kinetic energy region of UPS spectra (Figure 2c), the work function shifted upward from 4.90 to $4.77 \mathrm{eV}$ after EG treatment. Accordingly, the Fermi edge shifted gradually toward the higher binding energy as shown in the HOMO onset region of the UPS spectra (Figure 2d), implying that the doping level decreased slightly with EG treatment as found in the UV-VisNIR spectra. As a result, the significantly increased electrical conductivity of PTbT-Me:PSS was attributed to the removal of excess insulating PSS ${ }^{-}$surrounding around the PTbT backbone, which greatly enhanced the charge transport. ${ }^{13 \mathrm{~b}, 21}$ Notably, PTbT-Me:PSS showed much lower doping level than that in PTbT-Me:Tos, as concluded from the Fermi level of PTbT-Me:Tos overlapping with the valence band. ${ }^{14 \mathrm{f}}$

Atomic force microcopy was applied to investigate the morphology changes of PTbT-Me:PSS thin films. As shown in Figure 3a, the pristine PTbT-Me:PSS thin film exhibited a smooth surface with a roughness of $R_{\mathrm{q}}=3.27 \mathrm{~nm}$, which could be due to the excess of insulating and flexible PSS wrapping the conjugated PT $b \mathrm{~T}$ backbone and thus led to its low electrical conductivity as also found in pristine PEDOT: PSS. $^{20}$ After the EG treatment (Figure 3b), an obviously continuous domain, larger roughness of $R_{\mathrm{q}}=5.40 \mathrm{~nm}$, was found in the de-doped PTbT-Me:PSS films, which implied
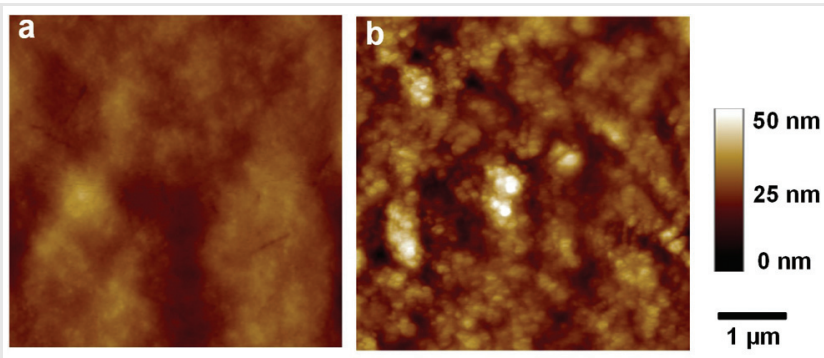

Figure 3 AFM images of (a) pristine and (b) EG-treated (30 min) PTbTMe:PSS films. 


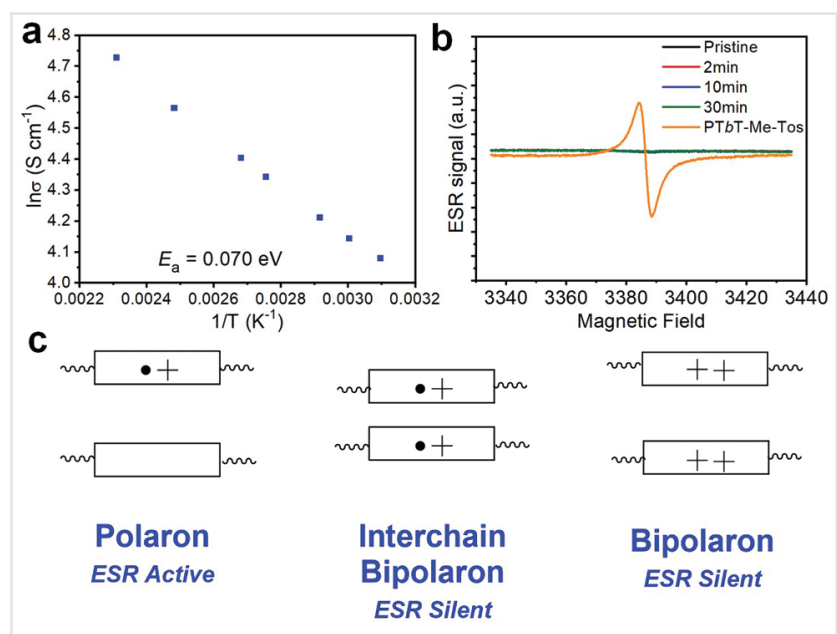

Figure 4 (a) Electrical conductivity changes of EG (30 min)-treated PTbT-Me:PSS film with temperature and (b) ESR spectra of pristine and EG-treated PTbT-Me:PSS film, and PTbT-Me:Tos film; (c) schematic diagrams illustrating the three types of charge carrier: polaron, interchain bipolaron, and bipolaron.

the changes of conformation after the removal of excess PSS and thus facilitated charge transport. ${ }^{20}$

To investigate the conduction mechanism of PTbT-Me: PSS, the electrical conductivity of EG-treated thin films were measured with changes in temperature. As shown in Figure 4a, the electrical conductivity increased linearly from 60 to $113 \mathrm{~S} \mathrm{~cm}^{-1}$ with heating, which can be attributed to the thermally activated hopping mechanism as previously found in PTbT-Me:Tos and PEDOT:PSS. ${ }^{13 c, 14 f, 22}$ After fitting to the Arrhenius equation: $\sigma=\sigma_{\mathrm{o}}\left[\mathrm{e}^{(-\mathrm{Ea} / R T)}\right]$, the activation energy $\left(E_{\mathrm{a}}\right)$ of EG-treated PTbT-Me:PSS was calculated to be $0.070 \mathrm{eV}$, which was much smaller than that of PTbT-Me:Tos $(0.29 \mathrm{eV})$ and was similar to that of the reported heavily doped PEDOT:PSS. A more efficient charge transport way might be formed in PTbT:PSS than in PTbT:Tos, which is consistent with the smaller activation energy. ${ }^{23}$

Different to the polaron-dominant PTbT-Me:Tos exhibiting a strong electron spin resonance (ESR) signal (paramagnetic), ${ }^{14 \mathrm{f}}$ no ESR signal was found in both pristine and EG-treated thin films of PTbT-Me:PSS and thus it was diamagnetic (Figure 4b). The relatively low doping level in PTbT-Me:PSS, as concluded from the UPS spectra, should contribute to a polaron-dominant charge carrier as shown in the previous report. ${ }^{16,22}$ However, the ESR measurement and the previous result seemed to contradict each other. A possible way should be that using PSS $^{-}$to replace Tos $^{-}$as a counterion coupled with a PTbT backbone may lead to the subtle energy changes including electrostatic interaction and lattice distortion. ${ }^{22}$ These changes may contribute to the stronger interchain interaction and thus interchain bipolaron is formed in PTbTMe:PSS (Figure 4c). Notably, in comparison to PEDOT, the same counterion that coupled to the conjugated backbone led to completely different types of charge carrier in PTbT as
Table 1 Charge carrier and conduction mechanism in PEDOT and PTbT with different counterions

\begin{tabular}{lllll}
\hline & PEDOT:PSS & PEDOT:Tos & PTbT-Me:PSS & PTbT-Me:Tos \\
\hline Charge carrier & Polaron & Bipolaron & $\begin{array}{l}\text { Interchain } \\
\text { bipolaron }\end{array}$ & Polaron \\
$\begin{array}{l}\text { Conduction } \\
\text { mechanism }\end{array}$ & Hopping & Semimetallic & Hopping & Hopping \\
\hline
\end{tabular}

summarized in Table 1. PEDOT:PSS was a polaron and showed thermally activated hopping conducting behavior. PEDOT-Tos's charge carrier was a bipolaron and showed metallic conductivity. In comparison, PTbT-Me:Tos was a polaron and a highly positive temperature-dependent electrical conductivity behavior was observed. Although the reason that led to the different kinds of charge carrier was unclear, further investigations on these polymers are important for the future application in organic spintronic and magnetic devices. As demonstrated before, bipolarondominant PEDOT:Tos showed much higher Seebeck coefficients and electrical conductivity than the polarondominant PEDOT:PSS. ${ }^{13 c, 24}$

Organic thermoelectric materials can directly transfer heat into electricity, which are promising in low/medium waste heat recycling, self-powered microelectronic devices etc., and thus have attracted great attention in the past two decades. ${ }^{25 a, 25 b, 25 c}$ Thermoelectric performance can be characterized by figure of merit: $Z T=\sigma S^{2} T / \mathrm{K}$, or power factor: $P F=\sigma S^{2}$, where $\sigma$ is the electrical conductivity, $S$ is the Seebeck coefficients, $\mathrm{K}$ is the thermal conductivity, and $T$ is the absolute temperature. In comparison with inorganic thermoelectric materials, organic conducting polymers normally hold low $\mathrm{K}\left(0.1-1 \mathrm{~W} \mathrm{~m}^{-1} \mathrm{~K}^{-1}\right)$, which makes organic conducting polymers a promising candidate in organic thermoelectrics at the low/medium temperature region. ${ }^{25}$ Therefore, we used this highly conductive PTbT-Me:PSS in thermoelectric application. The Seebeck coefficients were obtained by measuring the thermovoltages after providing a temperature difference on two sides of the films. As shown in Figure 5a, the Seebeck coefficients increased gradually with EG
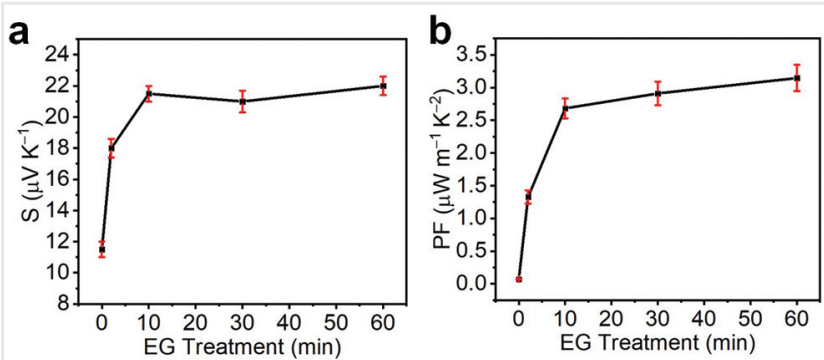

Figure 5 (a) Seebeck coefficients and (b) power factor changes of PTbT-Me:PSS films with EG treatment. 
treatment from 11.5 to $22 \mu \mathrm{V} \mathrm{K} \mathrm{K}^{-1}$, which was different from the commonly found negatively $S-\sigma$ dependent behavior. The simultaneously increasing Seebeck coefficients and electrical conductivity after EG treatment should be attributed to the removal of excess PSS, contributing to the significantly enhanced charge mobility, which overwhelmed the slightly decreased doping levels. ${ }^{13 \mathrm{~b}}$ As a result, the power factor of the PTbT-Me:PSS film was enhanced dramatically from 0.071 to $3.1 \mu \mathrm{W} \mathrm{m} \mathrm{m}^{-1}$ $\mathrm{K}^{-2}$ under ambient conditions, demonstrating that this polymer should be a good candidate for thermoelectric conversion.

\section{Conclusions}

In summary, we designed and prepared the waterdispersible conducting polymer PTbT-Me:PSS for organic electronics by the control of linear polymerization and using the large surfactant counterion $\mathrm{PSS}^{-}$. PTbT-Me:PSS thin films showed an electrical conductivity as high as 68 $S \mathrm{~cm}^{-1}$ under ambient conditions, after EG treatment. Intriguingly, in comparison with polaron-dominant PTbTMe:Tos, PTbT-Me:PSS showed an interchain bipolarondominant transport behavior. We applied this conducting polymer for thermoelectric application, in which a power factor of $3.1 \mu \mathrm{W} \mathrm{m} \mathrm{m}^{-1} \mathrm{~K}^{-2}$ at room temperature was obtained. Further optimization of the polymerization method and posttreatment should further enhance the electrical property. The combination of solution-processability and highly conductive property in PTbT-Me: PSS makes this quinoid-resonant conducting polymer very promising in the electronic market.

\section{Funding Information}

We thank the National Key Research and Development Program of China (No. 2019YFA0705900 and 2017YFA02 04701) funded by MOST and the National Natural Science Foundation of China (No. 21661132006, 21572234, and 91833304) for the financial support.

\section{Acknowledgments}

We thank Prof. Wei Xu and Prof. Daoben Zhu from ICCAS for the Seebeck coefficient measurements.

\section{Supporting Information}

Supporting information for this article is available online at http://doi.org/10.1055/s-0040-1714145.

\section{References}

(1) Heeger, A. J. Angew. Chem. Int. Ed. 2001, 40, 2591.

(2) (a) Shirakawa, H.; Louis, E. J.; MacDiarmid, A. G.; Chiang, C. K.; Heeger, A. J. J. Chem. Soc., Chem. Commun. 1977, 578. (b) Naarmann, H.; Theophilou, N. Synth. Met. 1987, 22, 1. (c) Park, Y. W. Synth. Met. 1991, 45, 173.

(3) (a) Cao, Y.; Treacy, G. M.; Smith, P.; Heeger, A. J. Synth. Met. 1993 57, 3526. (b) Toshima, N. Macromol. Symp. 2002, 186, 81. (c) Li, J.; Tang, X.; Li, H.; Yan, Y.; Zhang, Q. Synth. Met. 2010, 160, 1153.

(4) Yan, H.; Ohta, T.; Toshima, N. Macromol. Mater.Eng. 2001,286, 139.

(5) Kirchmeyer, S.; Reuter, K. J. Mater. Chem. 2005, 15, 2077.

(6) Chen, R.; Sun, K.; Zhang, Q.; Zhou, Y.; Li, M.; Sun, Y.; Wu, Z.; Wu, Y.; Li, X.; Xi, J.; Ma, C.; Zhang, Y.; Ouyang, J. iScience 2019, 12, 66.

(7) (a) Yano, H.; Kudo, K.; Marumo, K.; Okuzaki, H. Sci. Adv. 2019, 5, v9492. (b) Zotti, G.; Zecchin, S.; Schiavon, G.; Groenendaal, L. B. Macromol.Chem. Phys. 2002, 203, 1958. (c) Persson, K. M.; Karlsson, R.; Svennersten, K.; Loffler, S.; Jager, E. W.; Richter-Dahlfors, A.; Konradsson, P.; Berggren, M. Adv. Mater. 2011, 23, 4403.

(8) (a) Kawase, T.; Sirringhaus, H.; Friend, R. H.; Shimoda, T. Adv. Mater. 2001, 13, 1601. (b) Okuzaki, H.; Ishihara, M.; Ashizawa, S. Synth. Met. 2003, 137, 947.

(9) Gustafsson, J. C.; Liedberg, B.; Inganäs, O. Solid State Ionics 1994, 69, 145.

(10) Zhang, F.; Johansson, M.; Andersson, M. R.; Hummelen, J. C.; Inganäs, O. Adv. Mater. 2002, 14, 662.

(11) Burroughes, J. H.; Bradley, D. D. C.; Brown, A. R.; Marks, R. N.; Mackay, K.; Friend, R. H.; Burns, P. L.; Holmes, A. B. Nature 1990, 347, 539.

(12) Jonas, F.; Morrison, J. T. Synth. Met. 1997, 85, 1397.

(13) (a) Kim, B.; Shin, H.; Park, T.; Lim, H.; Kim, E. Adv. Mater. 2013, 25 , 5483. (b) Kim, G. H.; Shao, L.; Zhang, K.; Pipe, K. P. Nat. Mater. 2013, 12, 719. (c) Bubnova, O.; Khan, Z. U.; Wang, H.; Braun, S.; Evans, D. R.; Fabretto, M.; Hojati-Talemi, P.; Dagnelund, D.; Arlin,J. B.; Geerts, Y. H.; Desbief, S.; Breiby, D. W.; Andreasen, J. W.; Lazzaroni, R.; Chen, W. M.; Zozoulenko, I.; Fahlman, M.; Murphy, P. J.; Berggren, M.; Crispin, X. Nat. Mater. 2014, 13, 190. (d) Shi, W.; Zhao, T.; Xi, J.; Wang, D.; Shuai, Z. J. Am. Chem. Soc. 2015, 137, 12929. (e) Weathers, A.; Khan, Z. U.; Brooke, R.; Evans, D.; Pettes, M. T.; Andreasen, J. W.; Crispin, X.; Shi, L. Adv. Mater. 2015, 27, 2101.

(14) (a) Neef, C. J.; Brotherston, I. D.; Ferraris, J. P. Chem. Mater. 1999, 11, 1957. (b) Pomerantz, M.; Gu, X.; Zhang, S. X. Macromolecules 2001, 34, 1817. (c) Sotzing, G. A.; Lee, K. Macromolecules 2002, 35, 7281. (d) Lee, B.; Seshadri, V.; Sotzing, G. A. Langmuir 2005, 21, 10797. (e) Patra, A.; Wijsboom, Y. H.; Leitus, G.; Bendikov, M. Chem. Mater. 2011, 23, 896. (f) Yuan, D.; Liu, L.; Jiao, X.; Zou, Y.; McNeill, C. R.; Xu, W.; Zhu, X.; Zhu, D. Adv. Sci. 2018, 5, 1800947. (g) Zhang, C.; Zang, Y.; Gann, E.; McNeill, C. R.; Zhu, X.; Di, C. A.; Zhu, D. J. Am. Chem. Soc. 2014, 136, 16176. (h) Ren, L.; Liu, F.; Shen, X.; Zhang, C.; Yi, Y.; Zhu, X. J. Am. Chem. Soc. 2015, 137, 11294. (i) Liu, F.; Zhou, Z.; Zhang, C.; Vergote, T.; Fan, H.; Liu, F.; Zhu, X. J. Am. Chem. Soc. 2016, 138, 15523.

(15) (a) Liu, F.; Espejo, G. L.; Qiu, S.; Oliva, M. M.; Pina, J.; Seixas de Melo, J. S.; Casado, J.; Zhu, X. J. Am. Chem. Soc. 2015, 137, 10357. (b) Mishra, A.; Ma, C.Q.; Bäuerle, P. Chem. Rev. 2009, 109, 1141. (c) Zhang, C.; Zhu, X. Acc. Chem. Res. 2017, 50, 1342. (d) Yuan, D. Chem 2019, 5, 744. (e) Yuan, D.; Huang, D.; Rivero, S. M.; Carreras, A.; Zhang, C.; Zou, Y.; Jiao, X.; McNeill, C. R.; Zhu, X.; Di, C.A.; Zhu, D.; Casanova, D.; Casado, J. Chem 2019, 5, 964. (f) Ren, L.; Fan, H.; Huang, D.; Yuan, D.; Di, C. A.; Zhu, X. Chem. Eur. J. 2016, 22, 17136. (g) Ren, L.; Yuan, D.; Gann, E.; Guo, Y.; Thomsen, L.; McNeill, C. R.; Di, C.A.; Yi, Y.; Zhu, X.; Zhu, D. Chem. Mater. 
2017, 29, 4999. (h) Yuan, D.; Huang, D.; Zhang, C.; Zou, Y.; Di, C. A.; Zhu, X.; Zhu, D. ACS Appl. Mater. Interfaces 2017, 9, 28795. (i) Ren, L.; Yuan, D.; Zhu, X. Chem. Asian J. 2019, 14, 1717. (j) Yuan, D.; Guo, Y.; Zeng, Y.; Fan, Q.; Wang, J.; Yi, Y.; Zhu, X. Angew. Chem. Int. Ed. 2019, 58, 4958.

(16) Swager, T. M. Macromolecules 2017, 50, 4867.

(17) Synthesis of PTbT-Me:PSS: TbT-Me monomer, $\mathrm{Fe}_{2}\left(\mathrm{SO}_{4}\right)_{3}$, and $\mathrm{PSSH}$ were added to a round-bottomed flask in deionized water (15 mg of TbT-Me in $1 \mathrm{~mL}$ of $\mathrm{H}_{2} \mathrm{O}$ ) under ambient conditions. The mixture was vigorously stirred at room temperature in air for 2 days. The insoluble precipitate was leached by a filter membrane and a black solution was obtained as shown in Figure S1. The optimization details are shown in Figure S2.

(18) Aust, R. B.; Bentley, W. H.; Drickamer, H. G. J. Chem. Phys. 1964, 41, 1856.

(19) Park, T.; Park, C.; Kim, B.; Shin, H.; Kim, E. Energy Environ. Sci. 2013, 6, 788.

(20) Fan, Z.; Li, P.; Du, D.; Ouyang, J. Adv. Energy Mater. 2017, 7, 1602116.

(21) Kim, Y. H.; Sachse, C.; Machala, M. L.; May, C.; Müller-Meskamp, L.; Leo, K. Adv. Funct. Mater. 2011, 21, 1076.

(22) Bubnova, O.; Crispin, X. Energy Environ. Sci. 2012, 5, 9345.

(23) Schwarze, M.; Gaul, C.; Scholz, R.; Bussolotti, F.; Hofacker, A.; Schellhammer, K. S.; Nell, B.; Naab, B. D.; Bao, Z.; Spoltore, D.;
Vandewal, K.; Widmer, J.; Kera, S.; Ueno, N.; Ortmann, F.; Leo, K. Nat. Mater. 2019, 18, 242.

(24) Bubnova, O.; Khan, Z. U.; Malti, A.; Braun, S.; Fahlman, M.; Berggren, M.; Crispin, X. Nat. Mater. 2011, 10, 429.

(25) (a) Poehler, T. O.; Katz, H. E. Energy Environ. Sci. 2012, 5, 8110. (b) Zhang, Q.; Sun, Y.; Xu, W.; Zhu, D. Adv. Mater. 2014, 26, 6829. (c) Russ, B.; Glaudell, A.; Urban, J. J.; Chabinyc, M. L.; Segalman, R. A. Nat. Rev. Mater. 2016, 1, 16050. (d) Sun, Y.; Di, C. A.; Xu, W.; Zhu, D. Adv. Electron. Mater. 2019, 5, 1800825. (e) Zhang, J.; Song, G.; Qiu, L.; Feng, Y.; Chen, J.; Yan, J.; Liu, L.; Huang, X.; Cui, Y.; Sun, Y.; Xu, W.; Zhu, D. Macromol. Rapid Commun. 2018, 39, 1800283. (f) Kiefer, D.; Kroon, R.; Hofmann, A. I.; Sun, H.; Liu, X.; Giovannitti, A.; Stegerer, D.; Cano, A.; Hynynen, J.; Yu, L.; Zhang, Y.; Nai, D.; Harrelson, T. F.; Sommer, M.; Moule, A. J.; Kemerink, M.; Marder, S. R.; McCulloch, I.; Fahlman, M.; Fabiano, S.; Müller, C. Nat. Mater. 2019, 18, 149. (g) Wu, J.; Sun, Y.; Pei, W. B.; Huang, L.; Xu, W.; Zhang, Q. Synth. Met. 2014, 196, 173. (h) Yao, C. J.; Zhang, H. L.; Zhang, Q. Polymers 2019, 11, 107. (i) Zeng, Y. J.; Wu, D.; Cao, X. H.; Zhou, W. X.; Tang, L. M.; Chen, K. Q. Adv. Funct. Mater. 2020, 30, 1903873. (j) Lu, Y.; Wang, J-Y.; Pei, J. Chem. Mater. 2019, 31, 6412. (k) Kroon, R.; Mengistie, D. A.; Kiefer, D.; Hynynen, J.; Ryan, J. D.; Yu, L.; Müller, C. Chem. Soc. Rev. 2016, 45, 6147. (l) McGrail, B. T.; Sehirlioglu, A.; Pentzer, E. Angew. Chem. Int. Ed. 2015, 54, 1710. 